\title{
Prevalence of asymptomatic SARS-CoV-2 Infection in couples seeking in vitro fertilization treatment in Riyadh, July-November, 2020
}

\begin{abstract}
Background: After the start of the coronavirus disease (COVID-19) pandemic, assisted reproductive services were suspended and restarted in in vitro fertilization (IVF) clinics worldwide. In our center we implemented screening of all couples for COVID-19 the day before starting the ovulation cycle and before the ovum pick-up (OPU) procedure.
\end{abstract}

Objectives: Assess the prevalence of asymptomatic COVID-19 among couples undergoing in vitro fertilization (IVF).

Design: Cross-sectional and retrospective cohort study.

Setting: An IVF unit in Riyadh from July to November 2020.

Patients and methods: Patients and their partners were tested for severe acute respiratory syndrome coronavirus 2 (SARS-CoV-2) infection 24-48 hours before their initial visit to initiate the fertility cycle and again prior to the ovum pick-up visit.

Main outcome measures: Prevalence of asymptomatic SARS-CoV-2 infection.

Sample size: 508 couples.

Results: A total of 508 couples were tested. Of the 508 cycles of therapy, 24 (4.7\%) were cancelled, $16(3.14 \%)$ before the start of the cycle, and $8(1.66 \%)$ before ovum pick-up, because one or both partners in the couple tested SARS-CoV-2 positive. Before the start of the cycle, 3 patients and their partners both tested positive; 7 patients tested positive and their partners tested negative and 6 partners tested positive and the patient testing negative. In addition, on retesting 481 couples before OPU 3 patients and their partners both tested positive; 3 patients tested positive, and their partners tested negative; and 2 partners tested positive and the patients tested negative.

Conclusion: The prevalence of asymptomatic SARS-CoV-2 infection in couples seeking IVF was higher than that in the general population. Implementing a policy of screening couples for SARS-CoV-2 prior to IVF treatment, minimized the possibility of transmission of SARS-CoV-2 from patients to healthcare workers.

Keywords: SARS-CoV-2, COVID-19, asymptomatic infection, in vitro fertilization
Volume 12 Issue 5 - 202I

\author{
Amani Aldriweesh, Ali Hibshi, Fatimah \\ Abdulsaud, Khalid Arab Awartani \\ In Vitro Fertilization Unit, King Faisal Specialist Hospital \& \\ Research Centre, Riyadh, Kingdom of Saudi Arabia
}

\begin{abstract}
Correspondence: Khalid Arab Awartani, In Vitro Fertilization Unit, King Faisal Specialist Hospital \& Research Centre, Makkah Al Mukarramah Branch Rd,Al Mathar Ash Shamali, Riyadh 1 1 564, Kingdom of Saudi Arabia, Tel +966-50-54 I-5597, Email kawartani@kfshrc.edu.sa
\end{abstract}

Received: August 29, 202I | Published: September 14, 2021
Abbreviations: IVF, in vitro fertilization; OPU, ovum pick-up

\section{Introduction}

Coronavirus disease (COVID-19), caused by severe acute respiratory syndrome coronavirus 2 (SARS-CoV-2) was first described in December 2019 in Wuhan, China and subsequently declared a pandemic in March 2020. ${ }^{1}$ Between December 2019 and November 2020, there were over 57.8 million cases of COVID-19 and 1.3 million deaths, worldwide. In the Kingdom of Saudi Arabia (KSA), there were 35,503 cases of confirmed COVID-19 as of November 22, 2020 with 102,000 cumulative cases per million population. ${ }^{2}$ Since COVID-19 became pandemic, assisted reproductive technique (ART) treatments have been suspended and restarted in in vitro fertilization (IVF) clinics worldwide. ${ }^{3}$ Our unit in Riyadh performs approximately 1,500 IVF cycles per year, of which approximately 300 cycles are for pre-implantation genetic testing and 1,200 are for infertility. The ART service was suspended from April 2020 until the end of June 2020 due to the COVID-19 pandemic. As SARS-CoV-2 has an incubation period of 1 to 14 days, on resuming the service in July 2020, we applied a screening policy to test all couples for SARS-CoV-2 before starting the cycle. ${ }^{4}$ We were unable to find data on the prevalence of SARS-CoV-2 infection among asymptomatic individuals. We sought to determine the prevalence of SARS-CoV-2 infection among patients using the our IVF service to help to refine the screening protocol for detecting asymptomatic SARS-CoV-2 infection among patients undergoing IVF treatment. The objective of the study was to assess the prevalence of SARS-CoV-2 infection among couples undergoing IVF.

\section{Materials and methods}

This study included couples who underwent IVF at the IVF unit from July 2020 to the end of November 2020. Saudi couples with a history of infertility or genetic disease requiring IVF or preimplantation genetic testing were eligible for inclusion in the study.

All couples starting an IVF cycle were required to go through a screening process. Prior to their IVF appointment, patients were telephoned by staff from the telemedicine clinic and asked if they and/ or their partner had any COVID-19 symptoms or had been exposed 
to individuals with known or suspected COVID-19. Individuals with COVID-19 symptoms or history of contact with an individual with suspected or confirmed COVID-19 reported during the screening call before the appointment to start the IVF cycle, and those with a history of SARS-CoV-2 infection or COVID-19 within the previous 3 months were excluded.

If both members of the couple did not report symptoms of COVID-19 or possible exposure, they were required to attend a drive-through clinic where they had oropharyngeal or nasopharyngeal swabs collected for SARS-CoV-2 testing. The swabs were tested for SARS-CoV-2 using a real-time reverse-transcription polymerase chain reaction assay. Each patient and their partner were screened twice, first on the day before starting the cycle and again 24-48 hours before the ovum pick-up (OPU) visit. If the patient and/or their partner tested positive, the treatment was deferred. If both members of the couple were negative, the clinic staff used standard personal protective equipment with a surgical mask during consultations and while performing OPU procedures.

The clinic uses three protocols. We primarily use a gonadotropin releasing hormone $(\mathrm{GnRH})$ antagonist protocol with subcutaneous gonadotropins because of its convenience to the patient and in order to avoid multiple hospital visits to receive intramuscular injections. The long protocol entails taking a single GnRH agonist in early follicular phase of the previous menstrual cycle and the short protocol entails starting the GnRH agonist in early follicular of same cycle.

The primary outcome was SARS-CoV-2 infection on testing prior to the visit to start the cycle and SARS-CoV-2 infection during the treatment cycle was a secondary outcome. All study data were stored in a hospital password-protected computer without patient names. Patients were identified by the study number on the data collection sheet. The data were analyzed using SPSS 20 (IBM Corp, Armonk, NY, USA).

The study protocol was approved by our institution's research ethics committee. The study was conducted according to good clinical practice guidelines and hospital policy. The requirement for informed consent was waived.

\section{Results}

A total of 508 couples participated in the study. The patient selection and testing process is shown in Figure 1. The majority of patients $(71 \%)$ were aged 36 years or less with a median age of 34 years (Figure 2a), and the majority of their partners (71\%) were aged 40 years or less with a median age 38 years (Figure $2 b$ ). The majority of patients $(\mathrm{n}=354,69.7 \%)$ received the $\mathrm{GnRH}$ antagonist protocol; $114(22.4 \%)$ received the long protocol; $24(4.7 \%)$ received the short protocol; and $16(3.2 \%)$ cancelled prior to the start of IVF (Table 1).

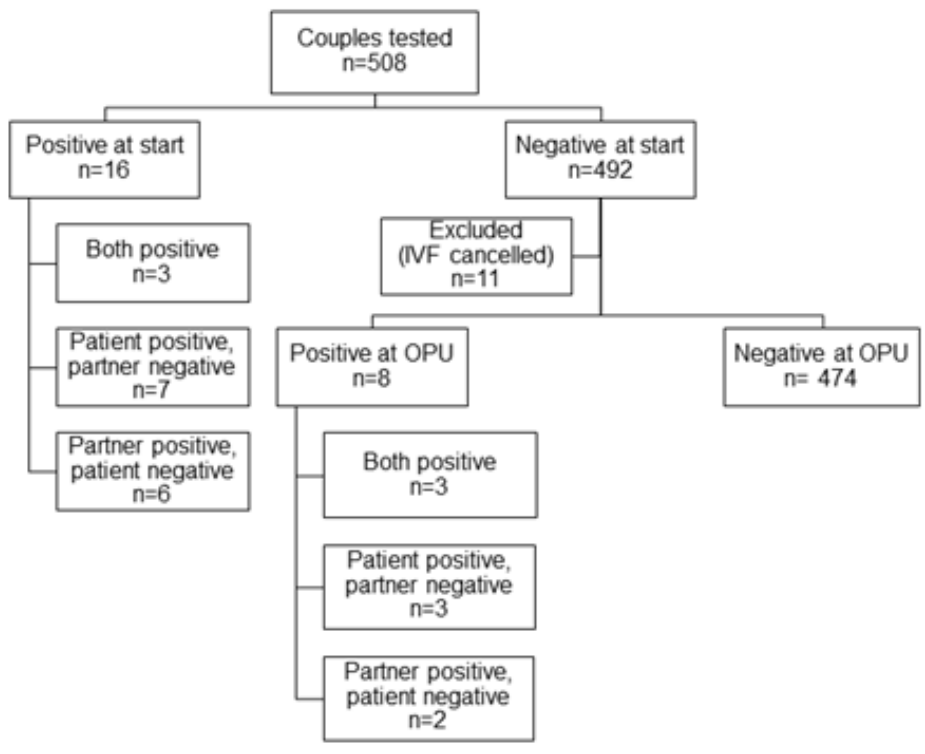

Figure I Flow chart of the screening process showing the number of in vitro fertilzation cycles deferred due to one or both members of the couple testing SARS-CoV-2 positive.
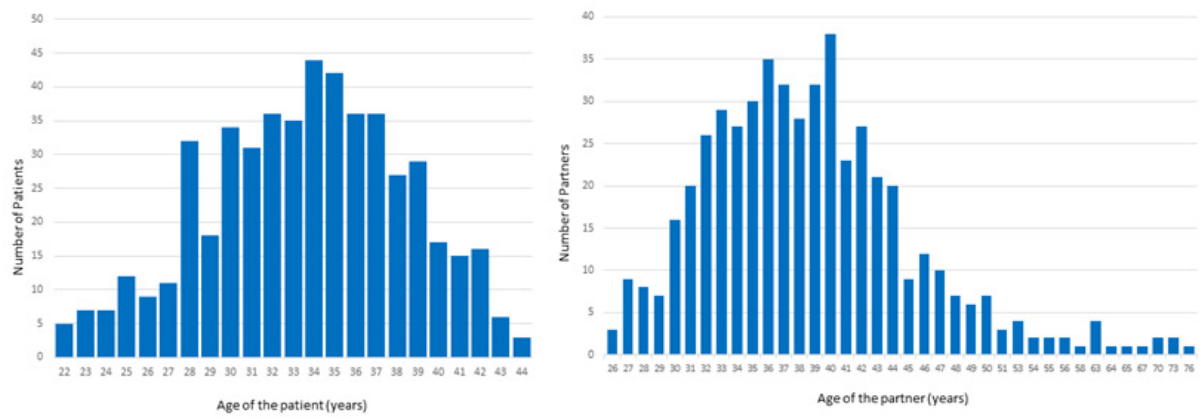

Figure 2 Age distribution of (a) the patients and (b) their partners. 
Table I In vitro fertilization treatment protocols used

\begin{tabular}{ll}
\hline Type of protocol & Number of patients (508) \\
\hline Antagonist & 354 \\
Long & $114(22.4 \%)$ \\
Short & $24(4.7 \%)$ \\
Cancelled at start & $16(3.2 \%)$
\end{tabular}

Out of the 508 patients scheduled to have IVF treatment, 11 IVF cycles were canceled for social reasons $(n=3)$, a lack of response after receiving the maximum dose of ovulation stimulation medication with lengthy stimulation $(\mathrm{n}=5)$ and the presence of ovarian cysts at the start of the cycle $(n=3)$. All 11 couples were negative on SARS-CoV-2 testing and were not retested because their visits had been cancelled (Table 2).

Table 2 Indication for cycle cancelation

\begin{tabular}{ll}
\hline Reason & Number of Patients (I I) \\
\hline Social reasons & 3 \\
Lack of response & 5 \\
Presence of ovarian cysts & 3
\end{tabular}

Out of the 508 patients scheduled to have IVF treatment, we cancelled a total 24 treatments $(4.7 \%), 16(3.14 \%)$ before starting the ovulation cycle, and $8(1.66 \%)$ before OPU because one or both members of the couple tested SARS-CoV-2 positive (Figure 1). Three couples tested positive at start of the cycle; and additional 7 patients tested positive with their partners testing negative and 6 partners tested positive with the patient testing negative. In addition, on retesting before OPU, 3 of the 481 tested positive; an additional 3 patients tested positive with their partners testing negative and 2 partners tested positive with the patients testing negative. Prior to the start of the cycle, of 1016 individuals tested, 19 (1.9\%) tested positive; on retesting prior to ovum pick-up, of the 962 individuals tested 11 $(1.1 \%)$ tested positive; and of the individuals tested positive as shown in Figure 3.

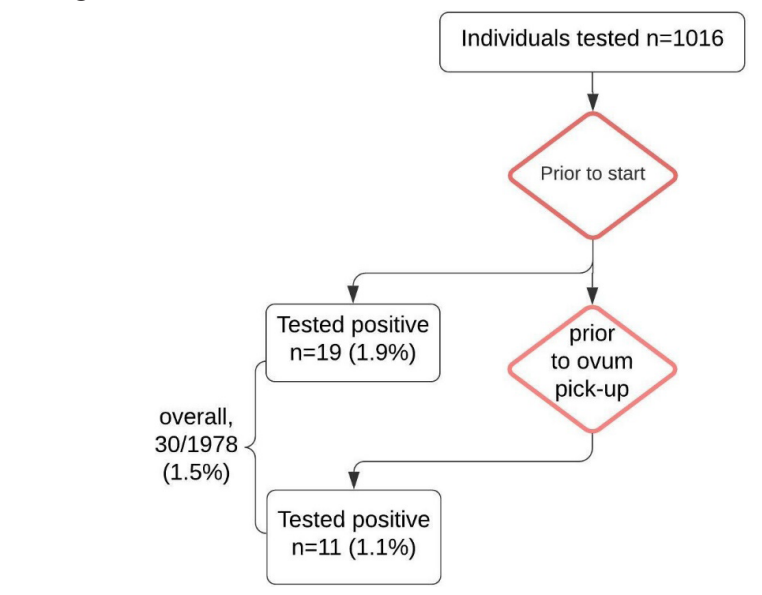

Figure 3 Number of indivituals tested SARS-CoV-2 positive in vitro fertilzation cycles.

\section{Discussion}

We determined the SARS-CoV-2 prevalence among couples seeking IVF who were not suspected to have COVID-19 because they were asymptomatic and did not report having contact with any individuals with COVID-19. The overall prevalence was higher than the estimated prevalence in the general population, which was approximately $1 \%$ at the highest of the COVID-19 epidemic in the KSA given that Saudi population 34,813,871 and the number of cases are $545367 .{ }^{5}$ Moreover, the positive rate per test at that period in Saudi Arabia were $0.6 \% .{ }^{6}$ Although those who tested positive were asymptomatic and had no known contact with anyone with COVID-19, the relatively high prevalence can be explained by the couples being mostly young and healthy, making them more likely to spend time outside their homes, thus having above average the amount of contact with other people. These mentioned results are supported by other studies were the proportion of asymptomatic infections among the included studies ranged from (1.4\%) to $(78.3 \%)$ and the need to assess the asymptomatic possibly infected from the general population still rise dilemma of cost-effective protocol. ${ }^{7}$ Furthermore the cost for health care system to obtain, perform and report the test were roughly estimated to be 750 in Saudi Riyals (SAR) (United States dollars (USD) 200) per person compared to hospitalization cost per patient (in SAR) was 48,436.18 $\pm 37,539.05$ ((USD) $12,914.08$ $\pm 10,008.68) .{ }^{8,9}$

Our approach of phone screening before the appointment, SARSCoV-2 testing prior to the start of the treatment cycle and again prior to the OPU, yielded valuable real-time data on the prevalence of SARS-CoV-2 infection in our patients and their partners. All our couples were followed-up in our unit and agreed to testing. They complied with symptom screening at every visit and universal use of face masks. Our hospital has a standard protocol for sample collection and testing using the SARS-CoV-2 PCR assay. Implementing the screening policy minimalized the possibility of patients and their partners transmitting SARS-CoV-2 to healthcare workers, including sonographers, physicians, and nurses, thus preventing staff shortages and possible temporary closures of the unit due to staff needing to self-isolate.

We found SARS-CoV-2 point prevalence in couples seeking IVF, who were not suspected to have COVID-19, to be higher than that in the general population. Implementing a screening policy minimalized the risk of SARS-CoV-2 transmission to healthcare workers.

\section{Acknowledgments}

None.

\section{Funding}

None.

\section{Conflicts of interest}

No potential conflicts of interest are reported by the authors.

\section{References}

1. Liu YC, Kuo RL, Shih SR. COVID-19: The first documented coronavirus pandemic in history. Biomed J. 2020;43(4):328-333.

2. World Health Organization. Weekly epidemiological update - 24 November 2020. 2021.

3. ESHRE COVID-19 Working Group, Vermeulen N, Ata B, et al. A picture of medically assisted reproduction activities during the COVID-19 pandemic in Europe. Hum Reprod Open. 2020;2020(3):hoaa035.

4. $\mathrm{Hu} \mathrm{B}$, Guo $\mathrm{H}$, Zhou $\mathrm{P}$, et al. Characteristics of SARS-CoV-2 and COVID-19. Nat Rev Microbiol. 2021;19(3):141-154.

5. World Health Organization. COVID-19 Explorer-September 9, 2021.

6. Hannah Ritchie, Edouard Mathieu, Lucas Rodés-Guirao, et al. Coronavirus Pandemic (COVID-19). 2020. 
7. Alene M, Yismaw L, Assemie MA, et al. Magnitude of asymptomatic COVID-19 cases throughout the course of infection: A systematic review and meta-analysis. PLoS One. 2021;16(3):e0249090.

8. Brooks ZC, Das S. COVID-19 Testing. Am JClin Pathol. 2020;154(5):575584.
9. Anas A, Yazed AlRuthia, Bander Balkhi, et al. Survival and estimation of direct medical costs of hospitalized COVID-19 patients in the kingdom of Saudi Arabia. Int J Environ Res Public Health. 2020;17(20):7458. 